\title{
Eficacia de los antihelmínticos para las parasitosis frecuentes
}

Efficacy of anthelmintics for frequent parasitosis

\section{Comentado de:}

Moser W. y Col. BMJ 2017;358:j4307. PMID: $28947636^{1}$

\section{Objetivos}

Evaluar la eficacia de los medicamentos contra los geohelmintos en términos de tasa de curación y tasa de reducción de huevos.

\section{Fuente de datos}

Se realizaron búsquedas en PubMed, ISI Web of Science, Embase, ScienceDirect, el Registro Central de Ensayos Clínicos Cochrane y la base de datos de la biblioteca de la Organización Mundial de la Salud desde 1960 hasta el 31 de diciembre de 2016. La búsqueda no tuvo limitaciones por idioma.

\section{Selección de estudios}

Ensayos controlados aleatorizados que evaluaron la eficacia de un régimen de dosis única de albendazol, mebendazol, levami- sol o pamoato de pirantel contra Ascaris lumbricoides, anquilos toma (Necator americanus y Ancylostoma duodenale) y Trichuris trichiura. Los resultados primarios incluyeron la tasa de curación analizada mediante un metaanálisis en red con modelos de regresión logística mixta y la tasa de reducción de huevos, con modelos lineales mixtos.

\section{Extracción de datos}

Dos revisores independientes revisaron los títulos y los resúmenes para detectar los estudios potencialmente elegibles. Cuando los artículos cumplieron con los criterios de inclusión, se examinaron todos los manuscritos y, para los ensayos incluidos, los mismos revisores extrajeron los datos de forma independiente.

\section{Resultados principales}

Ver la Tabla 1 para un resumen de los resultados principales.

Tabla 1. Curación y reducción de huevos posterior al tratamiento farmacológico. Notas: ${ }^{*} P<0.05$, ${ }^{* \star} P<0.01$, ${ }^{* \star \star} P<0.001$ para comparación con placebo. IC95\%: Intervalo de Confianza del $95 \%$

\begin{tabular}{|c|c|c|c|}
\hline Parásito & Agente & $\begin{array}{l}\text { Curación, } \\
\qquad \%(\text { IC } 95 \%)\end{array}$ & $\begin{array}{l}\text { Reducción de los huevos } \\
\qquad \% \text { (IC } 95 \%)\end{array}$ \\
\hline A. lumbricoide & $\begin{array}{l}\text { Albendazol } \\
\text { Mebendazol } \\
\text { Levamisol } \\
\text { Pamoato de pirantel }\end{array}$ & $\begin{array}{l}95,7^{* \star *}(93,2 \text { a } 97,3) \\
96,2^{* * *}(92,3 \text { a } 98,1) \\
97,3^{* * *}(84,2 \text { a } 99,6) \\
92,6^{* * *}(85,6 \text { a } 96,3)\end{array}$ & $\begin{array}{l}98,5^{\star \star \star}(94,9 \text { a } 100,0) \\
98,0^{\star \star \star}(94,0 \text { a } 100,0) \\
96,4^{\star \star \star}(82,3 \text { a } 100,0) \\
94,3^{\star \star \star}(88,3 \text { a } 100,0)\end{array}$ \\
\hline Anquilostoma & $\begin{array}{l}\text { Albendazol } \\
\text { Mebendazol } \\
\text { Levamisol } \\
\text { Pamoato de pirantel }\end{array}$ & $\begin{array}{c}79.5^{\star * \star}(71,5 \text { a } 85,6) \\
32,5^{\star}(20,8 \text { a } 46,9) \\
10,3(2,4 \text { a } 35,2) \\
49,8^{\star *}(29,5 \text { a } 70,1)\end{array}$ & $\begin{array}{c}89,6^{\star * *}(81,9 \text { a } 97,3) \\
61,0^{\star \star *}(52,0 \text { a } 69,9) \\
61.8^{*}(30,3 \text { a } 93,3) \\
71,9^{\star * *}(54,7 \text { a } 89,0)\end{array}$ \\
\hline T. trichiura & $\begin{array}{l}\text { Albendazol } \\
\text { Mebendazol } \\
\text { Levamisol } \\
\text { Pamoato de pirantel }\end{array}$ & $\begin{array}{c}30,7^{\star * *}(21,0 \text { a } 42,5) \\
42,1^{* * *}(25,9 \text { a } 60,2) \\
29,5(6,1 \text { a } 72,9) \\
20,2(7,3 \text { a } 44,7)\end{array}$ & $\begin{array}{c}49,9^{\star * *}(39,0 \text { a } 60,6) \\
66,0^{\star * *}(54,6 \text { a } 77,3) \\
28,3(6,7 \text { a } 49,8) \\
47,5^{\star *}(25,5 \text { a } 69,6)\end{array}$ \\
\hline
\end{tabular}

\section{Conclusiones}

Los cuatro fármacos antihelmínticos investigados mostraron una superioridad muy significativa sobre el placebo. Si bien solo el albendazol mostró una buena eficacia contra la infección por anquilostomas, todos los fármacos tuvieron una eficacia baja frente a T. trichiura. La disminución de la eficacia de albendazol contra $T$. trichiura en las últimas dos décadas es preocupante. 


\section{Comentario}

Las parasitosis por geohelmintos son un problema de salud pública a nivel mundial, especial mente en poblaciones de escasos recursos económicos, donde son una importante fuente de morbilidad y mortalidad ${ }^{2}$. Tienen un mayor impacto en niños y niñas en edad preescolar, escolar y embarazadas, afectando a las futuras generaciones y la productividad económica del país. El uso de antihelmínticos no se debe limitar al de la infección sintomática, sino que se recomienda el tratamiento general de poblaciones con alta endemicidad. Cuando la prevalencia en un área determinada es mayor de $50 \%$, la OMS recomienda tratamiento bi o trianual en grupos escolares o de riesgo, y si está entre un 20 a $50 \%$ lo recomienda anualmente ${ }^{3}$. En la Argentina la prevalencia es variable entre el 0 y el $88,9 \%{ }^{2}$, con una gran heterogeneidad. Un estudio que incluyó ocho provincias arrojó que Salta, Misiones y Santa Fe (y posiblemente también la ciudad de La Plata) tienen prevalencias mayores al $20 \%$, lo cual justificaría campañas de desparasitación masivas. La frecuente resistencia de $T$. trichiura orientaría al uso de terapias combinadas con distintos fármacos, incluyendo albendazol o mebendazol junto a ivermectina ${ }^{4}$. Argentina ha contado con un Programa Nacional de Desparasitación Masiva, focalizado en áreas de mayor prevalencia y en población de 2 a 14 años ${ }^{5}$.

\section{Conclusiones del comentador}

Los fármacos usados para el tratamiento de geohelmintos siguen siendo efectivos contra algunos parásitos, pero preocupa el aumento de la resistencia en algunos de ellos. Se requiere continuar con campañas de desparasitación masiva en población vulnerable, orientadas por estudios de prevalencia. El uso de terapias combinadas podría ser recomendable.

Gustavo Andrés Castro Torres [ Universidad de Antioquia, Facultad de Medicina gustavoandresc@gmail.com ]

Castro Torres GA Eficacia de los antihelmínticos para las parasitosis frecuentes. Evid Actual Pract Ambul. 2019;22(3):e001106. Comentado de: Moser W, Schindler C, Keiser J. Efficacy of recommended drugs against soil transmitted helminths: systematic review and network meta-analysis. BMJ. 2017 Sep 25;358:j4307. PMID: 28947636

\section{Referencias}

1. Moser W, Schindler C, Keiser J. Efficacy of recommended drugs against soil transmitted helminths: Systematic review and network meta-analysis. BMJ (Online). 2017;358. Available from: 10.1136/bmj.j4307.

2. Socías ME. Geohelmintiasis en la Argentina: Una revisión sistemática. Medicina (B Aires). 2014;74(1):29-36. Available from: https:// medicinabuenosaires.com/revistas/vol74-14/n1/29-36-Med5-6079-Socias.pdf.

3. WHO. Preventive Chemotherapy in Human Helminthiasis: Coordinated Use of Anthelminthic Drugs in Control Interventions. A Manual for Health Professionals and Programme Managers. Geneva: World Health Organization; 2006. Available from: https://apps.who.int/iris/bitstream/handle/10665/ 43545/9241547103_eng.pdf;jsessionid=9F044AC5A9FBE17162CB8B9781CB04C0?sequence=1.

4. Knopp S, Mohammed KA, Speich B, et al. Albendazole and mebendazole administered alone or in combination with ivermectin against Trichuris trichiura: a randomized controlled trial. Clin Infect Dis. 2010;51(12):1420-8. Available from: 10.1086/657310.

5. Fernández-Prieto EA. Evaluación de impacto del programa nacional de desparasitación masiva - Argentina . Rio de Janeiro, Brasil; 2010. Available from: http://bases.bireme.br/cgi-bin/wxislind.exe/iah/online/?IsisScript=iah/iah.xis\&src=google\&base=LILACS\&lang=p\&nextAction=Ink\&exprSearch= 587482\&indexSearch $=$ ID. 